\title{
Breaking the symmetries in self-induced flavor conversions of neutrino beams from a ring
}

\author{
Alessandro Mirizzi ${ }^{1,2}$ \\ ${ }^{1}$ Dipartimento Interateneo di Fisica "Michelangelo Merlin", Via Amendola 173, 70126 Bari, Italy \\ ${ }^{2}$ Istituto Nazionale di Fisica Nucleare - Sezione di Bari, Via Amendola 173, 70126 Bari, Italy
}

\begin{abstract}
Self-induced flavor conversions of supernova (SN) neutrinos have been characterized in the spherically symmetric "bulb" model, reducing the neutrino evolution to a one dimensional problem along a radial direction. We lift this assumption, presenting a two-dimensional toy-model where neutrino beams are launched in many different directions from a ring. We find that self-interacting neutrinos spontaneously break the spatial symmetries of this model. As a result the flavor content and the lepton number of the neutrino gas would acquire seizable direction-dependent variations, breaking the coherent behavior found in the symmetric case. This finding would suggest that the previous results of the self-induced flavor evolution obtained in one-dimensional models should be critically re-examined.
\end{abstract}

PACS numbers: 14.60.Pq, 97.60.Bw

\section{INTRODUCTION}

Dense neutrino gases in early universe or emitted from core-collapse supernovae ( $\mathrm{SNe}$ ) represent unique cases to probe the effect of the neutrino-neutrino interactions on the flavor conversions. Indeed, in these environments the neutrino-neutrino interactions would generate a large neutrino potential $\mu \sim \sqrt{2} G_{F} n_{\nu}$ that in some cases can exceed the ordinary matter term $\lambda=\sqrt{2} G_{F} n_{e}$ and the neutrino vacuum oscillation frequency $\omega=\Delta m^{2} / 2 E$. When this situation is encountered the neutrino-neutrino potential would dominate the flavor evolution producing large self-induced flavor conversions (see [1] for a review). A vivid activity on these effects in the context of SN neutrinos has flourished since a decade [2 5]. Indeed, it has been realized that in the deepest $\mathrm{SN}$ regions self-induced effects can produce collective neutrino oscillations, leading to peculiar spectral features in the oscillated neutrino spectra, dubbed as spectral swaps and splits [6 10].

The development of the self-induced flavor conversions is associated with instabilities in the flavor space that are triggered by the interacting neutrinos. The first one to be noticed was the bimodal instability present even in an homogeneous and isotropic neutrino gas [4]. In particular, it was shown that an ensemble initially composed of equal densities of $\nu_{e}$ and $\bar{\nu}_{e}$ in the presence of a dominant neutrino-neutrino interaction term would exhibit in inverted mass hierarchy $\left(\Delta m^{2}<0\right)$ large pair-conversions of the type $\nu_{e} \bar{\nu}_{e} \leftrightarrow \nu_{x} \bar{\nu}_{x}$ even with a small mixing angle. This behavior has been explained in terms of an unstable pendulum in flavor space, where the instability is associated with the tiny mixing angle [4, 11]. Furthermore, it has been shown that if one introduces an anisotropy in the neutrino gas, this can dramatically change the previous solution. Indeed, in a non-isotropic neutrino ensemble, the neutrino-neutrino interaction term contains multi-angle effects since the current-current nature of the low-energy weak interactions introduces an angle dependent term $\left(1-\mathbf{v}_{\mathbf{p}} \cdot \mathbf{v}_{\mathbf{q}}\right)$ between two interacting neutrino modes [3, 12]. In the case of a gas completely symmetric in flavor content of $\nu$ and $\bar{\nu}$, even a small deviation from a perfect isotropy is enough to produce a multiangle dechoerence leading to a flavor equilibrium among the different neutrino species in both the mass hierarchies [13]. Multi-angle effects have been extensively studied in the context of flavor evolution of SN neutrinos [14], whose emission is far from isotropic. It has been realized that in some cases they can destroy the collective behavior of the flavor evolution observed in an isotropic environment [13, 15, 16]. Multi-angle effects can also lead to a trajectory-dependent matter term, which if strong enough suppresses the self-induced conversions [17 20]. In the context of SN neutrinos it has been often assumed an axially symmetric neutrino emission in oder to integrate out the azimuthal angle in the multi-angle kernel. However, it has been found that lifting this assumption, neutrino-neutrino interactions can break axial symmetry and lead to azimuthal-angle dependent flavor conversions 21 27].

The lesson that has been gained from these situations is that self-interacting neutrinos can spontaneously break the symmetries of the initial conditions, since small deviations from them can be dramatically amplified during the further flavor evolution. This insight has recently stimulated doubts about the validity of the solution of the SN neutrino equations of motion worked out in the so-called "bulb model" 3, 5, 16]. In this framework it is assumed the spherical symmetry about the center of the $\mathrm{SN}$ and the axial symmetry about any radial direction. These two symmetries allow one to reduce the problem to a one-dimensional evolution along a radial direction. Remarkably, removing the assumption of spherical symmetry it would necessary to solve a challenging multidimensional problem to characterize the neutrino flavor evolution.

In this context, in order to show how deviations from the spatial symmetries of a system would affect the flavor evolution a simple two-dimensional model has been recently proposed in 28]. Namely, monochromatic neu- 
trinos streaming in a stationary way in two directions ("left" $L$ and "right" $R$, respectively) from an infinite boundary plane at $z=0$ with periodic conditions on $x$ and translation invariance along the $y$ direction. Remarkably, there is a correspondence between the symmetries of the bulb model and the ones of this planar case. Indeed, the translational symmetry in the $x$ direction in the planar model corresponds to the spherical symmetry of the bulb-model and the $L-R$ symmetry is equivalent to the axial symmetry in the spherical case. By means of a stability analysis of the linearized equations of motion, it has been shown in the planar model that if one perturbs the initial symmetries of the flavor content in both the two emission modes and along the boundary in the $x$ direction, then self-induced oscillations can spontaneously break both these spatial symmetries [28]. In 29] we have recently performed a numerical study of the flavor evolution for this case. We found that the initial small perturbations are amplified by neutrino interactions, leading to non-trivial two-dimensional structures in the flavor content and lepton number of the neutrino enseble, that would exhibit large space fluctuations.

The purpose of this paper is to develop a twodimensional model to capture more closely some of the features of the SN environment. In particular, with respect to the planar model considered in [28, 29] we make the following improvements: (i) $\nu$ emission from a ring mimicking the neutrino-sphere, (ii) parameters inspired by the SN neutrino emissivity, (iii) declining neutrino density from the boundary, (iv) multi-angle effects. We also assume that self-induced flavor conversions would develop without any hindrance caused by a large matter term. Perturbing the neutrino emission in the translational symmetry on the ring and in the emission directions, we find the spontaneous breaking of these symmetries in both normal and inverted mass hierarchies. As a consequence the flavor content and the lepton number of the neutrino ensemble acquires seizable variations along different lines of sight. These findings are presented as follows. In Sec. II we describe the features of our two-dimensional model. We discuss the equations of motion to characterize the two-dimensional flavor evolution. We show how it is possible to solve this problem by Fourier transforming these equations, obtaining a tower of ordinary differential equations for the different Fourier modes. In Sec. III we present the numerical results of our study. We show how the breaking of the spatial symmetries produce direction-dependent variations in the flavor content of the ensemble. Finally in Sec. IV we discuss about future developments and we conclude.

\section{TWO-DIMENSIONAL MODEL}

\section{A. Equations of motion}

Characterizing the SN neutrino flavor dynamics amounts to follow the spatial evolution of the neutrino fluxes. For a stationary neutrino emission, the Equations of Motion (EoMs) of the $\nu$ space-dependent occupation numbers $\varrho(\mathbf{r}, \mathbf{p})$ with momentum $\mathbf{p}$ at position $\mathbf{r}$ are [31, 32]

$$
\mathbf{v} \cdot \nabla_{\mathbf{r}} \varrho=-i[\Omega, \varrho]
$$

where we indicate with sans-serif vectors in flavor space, while for the ones in real space we use the bold-face. At the left-hand-side of Eq. (11) there is the Liouville operator representing the drift term proportional to the neutrino velocity $\mathbf{v}$, due to particle free streaming. Note that we are neglecting external forces and an explicit time dependence of the occupation numbers. On the right-handside of Eq. (11) the matrix $\Omega$ is the full Hamiltonian that reads

$\Omega=\frac{\mathrm{M}^{2}}{2 E}+\sqrt{2} G_{F}\left[\mathrm{~N}_{l}+\int_{-\infty}^{+\infty} d E^{\prime} E^{\prime 2} \int \frac{d \mathbf{v}^{\prime}}{(2 \pi)^{3}} \varrho^{\prime}\left(1-\mathbf{v} \cdot \mathbf{v}^{\prime}\right)\right]$,

where $\mathrm{M}^{2}$ is the matrix of the mass-squared, responsible of the vacuum oscillations. The ordinary matter effects on neutrino flavor conversions is accounted by the matrix of charged lepton densities $N_{l}$. Finally, the neutrinoneutrino interaction potential is represented by the last term of the right-hand-side, where the integral in $d \mathbf{v}^{\prime}$ is on the unit sphere and the occupation numbers $\varrho^{\prime}$ depend on $\mathbf{r}, E^{\prime}, \mathbf{v}^{\prime}$. Note that we use negative $E$ to denote antineutrinos.

In order to show the effect of spontaneous breaking of spatial symmetries we consider them to be emitted in a plane from a ring with radius $r=R$. We have then a two-dimensional model for which it is natural to use a system of polar coordinates to describe the neutrino position vector $\mathbf{r}=(r, \phi)$ where $r$ is the radius, $\phi \in[0 ; 2 \pi]$ is the polar angle, as shown in Fig. 1.

The neutrino velocity can be decomposed in the radial $\left(v_{r}\right)$ and transverse $\left(v_{t}\right)$ component defined as $\mathbf{v}=$ $\left(v_{r}, v_{ \pm}\right)=\left(\cos \theta_{r}, \pm \sin \theta_{r}\right)$, where $\theta_{r} \in[0, \pi / 2]$ is the angle between the radial direction and the one of the neutrino propagation (see, e.g., [33]), and the \pm sign indicate a transverse velocity in the clock-wise $\left(v_{+}\right)$or anti-clockwise $\left(v_{-}\right)$direction with respect to the radial direction, respectively. We mention that in the recent multi-angle study [34], where neutrinos emitted from a plane were considered, the range in the emission angles was $\theta \in[0, \pi]$. With this choice it is not necessary to distinguish clock-wise or anti-clockwise modes. However, in our work we preferred to use have the $\theta_{r} \in[0, \pi / 2]$ in order to start with a situation symmetric in the two emission directions \pm and show the effect of breaking of this discrete symmetry. Note that the local angle $\theta_{r}$ would depend on the radius $r$. In order to avoid this effect, in the literature it is preferred to label the neutrino modes in terms of their emission angle $\vartheta_{R} \in[0, \pi / 2]$ along the boundary at $r=R$. The two angles $\theta_{r}$ and $\theta_{R}$ are related by [16]

$$
R \sin \theta_{R}=r \sin \theta_{r}
$$




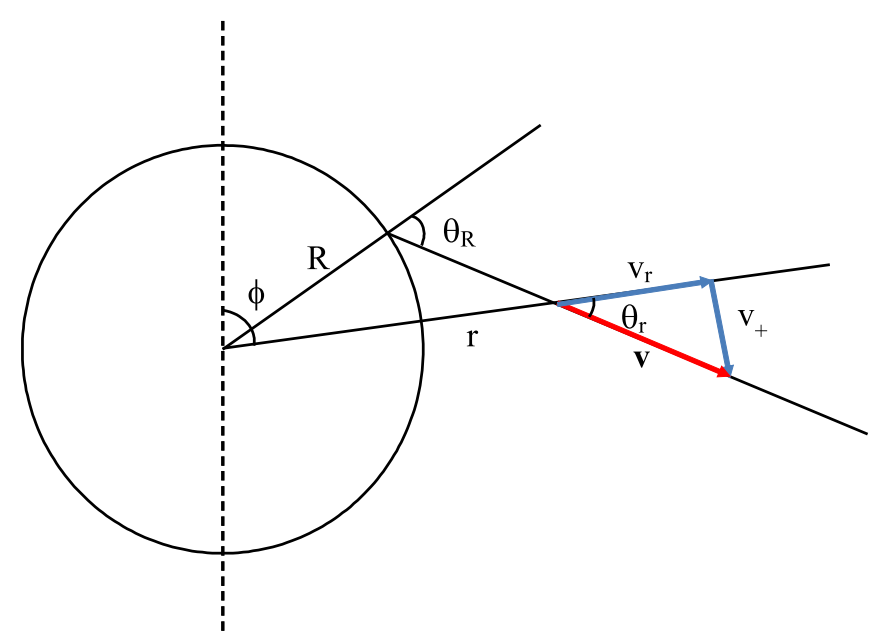

FIG. 1: Two-dimensional model for the neutrino beams emitted from a ring with radius $r=R$.

Furthermore, we introduce the angular variable $u=$ $\sin \theta_{R}, u \in[0,1]$. With this choice the components of the neutrino velocity are [21]

$$
\begin{gathered}
v_{r}=\cos \theta_{r}=\sqrt{1-\frac{R^{2}}{r^{2}} u^{2}}, \\
v_{t} \equiv v_{ \pm}= \pm \sin \theta_{r}= \pm \frac{R}{r} u .
\end{gathered}
$$

We assume that the neutrino distributions at $r=R$ in the energy $E$ and in the angular variables $u$ and in the $R, L$ directions can be factorized as

$$
F_{ \pm}(E, u)=F_{\nu}(E) \times F_{\nu}(u) \times F_{ \pm} .
$$

We assume the neutrino angular distributions to be flat in $u$ and equal for all the flavors, i.e. $F_{\nu}(u)=1$.

Concerning the distributions in the clock-wise $(+)$ or anti-clockwise $(-)$ directions, we assume that these are given by

$$
F_{ \pm}=\frac{\left(1+\beta_{ \pm}\right)}{2+\beta_{+}+\beta_{-}}
$$

where the quantities $\beta_{ \pm} \ll 1$ are introduced to slightly perturb the \pm of a given $u$ mode at the boundary.

The neutrino number flux $F_{\nu}(E)$ at the ring is given by

$$
F_{\nu}(E)=\frac{1}{4 \pi R^{2}} \frac{L_{\nu}}{\left\langle E_{\nu}\right\rangle} f_{\nu}(E),
$$

where we have normalized the neutrino emission on a sphere with radius $R$.

\section{B. Two-flavor case}

In the following we will consider only a two-flavor system $\left(\nu_{e}, \nu_{x}\right)$ where $x=\mu, \tau$ and we will describe the neutrino energy modes in terms of the two neutrino frequency $\omega=\Delta m^{2} / 2 E_{0}$, where $\Delta m^{2}=m_{2}^{2}-m_{1}^{2}$ is the mass-squared difference. We have assumed a monochromatic neutrino emission with $E=E_{0}$. In the two-flavor case the density matrices are projected over the Pauli matrices $\sigma$ obtaining the polarization vectors in the usual way [16], where we normalize the (anti)neutrino polarization vectors to the difference of the anti-neutrino fluxes at the boundary.

The Liouville operator on the left-hand-side of the EoMs [Eq. (1)] assumes the form

$$
\mathbf{v} \cdot \nabla_{\mathbf{r}}=v_{r} \frac{d}{d r}+\frac{v_{ \pm}}{r} \frac{d}{d \phi},
$$

so that the EoMs read (see also [21])

$$
\begin{aligned}
\frac{d}{d r} \mathrm{P}_{ \pm, u} & =-\frac{v_{ \pm}}{v_{r} r} \frac{d}{d \phi} \mathrm{P}_{ \pm, u} \\
& +\left[\frac{\omega}{v_{r}} \mathrm{~B}+\Omega_{ \pm}^{\nu \nu}\right] \times \mathrm{P}_{ \pm, u},
\end{aligned}
$$

where we indicated with sans-serif the vectors in flavor space. In particular, the unit vector $B=\left(B^{1}, B^{2}, B^{3}\right)$ points in the mass eigenstate direction in flavor space, such that $B \cdot e_{3}=-\cos \vartheta$, where $\vartheta$ is the vacuum mixing angle. For simplicity we neglect a possible matter effect, assuming that its only role would be to reduce the effective in-medium mixing angle, $\vartheta \ll 1[4]$. The neutrino-neutrino interaction terms has a multi-angle kernel $\left(1-\mathbf{v}_{\mathbf{p}} \cdot \mathbf{v}_{\mathbf{q}}\right)$ which takes the form

$$
\begin{aligned}
& \frac{1}{v_{r}} \int d \theta_{r}^{\prime}\left[1-v_{r} v_{r}^{\prime}-v_{t} v_{t}^{\prime}\right] \mathrm{D}^{\prime} \\
= & \frac{R}{r} \int d \vartheta_{R}^{\prime} \cos \vartheta_{R}^{\prime}\left[\frac{1}{v_{r} v_{r}^{\prime}}-1-\frac{v_{t} v_{t}^{\prime}}{v_{r} v_{r}^{\prime}}\right] \mathrm{D}^{\prime}
\end{aligned}
$$

where $\mathrm{D}$ is the difference between the neutrino and antineutrino polarization vector of a given mode, and we used from Eq. (3)

$$
d \theta_{r}=\frac{R}{r} \frac{\cos \vartheta_{R}}{\cos \theta_{r}} d \vartheta_{R}=\frac{R}{r} \frac{\cos \vartheta_{R}}{v_{r}} d \vartheta_{R}=\frac{R}{r} \frac{d u}{v_{r}} .
$$

In the large-distance limit $(r \gg R)$ one can expand Eq. (10) obtaining

$$
\frac{1}{2} \frac{R}{r} \int d u^{\prime}\left[v_{t}-v_{t}^{\prime}\right]^{2} \mathrm{D}^{\prime}
$$

We note that for the case we are studying the selfinteraction term declines as $r^{-3}$, while in the SN case it declines as $r^{-4}$. Considering the contribution of the clockwise $(+)$ and anti-clockwise $(-)$ modes in the previous equation one gets

$$
\begin{aligned}
v_{t}^{2} \mathrm{D}^{\prime} & =\left(\frac{R}{r}\right)^{2} u^{2}\left(\mathrm{D}_{+, u^{\prime}}+\mathrm{D}_{-, u^{\prime}}\right) \\
v_{t} v_{t}^{\prime} \mathrm{D}^{\prime} & =\mp\left(\frac{R}{r}\right)^{2} u u^{\prime}\left(\mathrm{D}_{+, u^{\prime}}-\mathrm{D}_{-, u^{\prime}}\right) .
\end{aligned}
$$


Then, the neutrino self-interaction term in the large distance limit $r \gg R$ assumes the form

$$
\begin{aligned}
\Omega_{ \pm}^{\nu \nu} & =\mu_{r} \int_{0}^{1} d u^{\prime}\left[\left(u^{2}+u^{\prime 2}\right) \frac{\left(\mathrm{D}_{+, u^{\prime}}+\mathrm{D}_{-, u^{\prime}}\right)}{2}\right. \\
& \left.\mp u u^{\prime}\left(\mathrm{D}_{+, u^{\prime}}-\mathrm{D}_{-, u^{\prime}}\right)\right]
\end{aligned}
$$

where the $\mp$ refers to the \pm modes respectively, and

$$
\begin{aligned}
\mu_{r} & =\left[F_{\bar{\nu}_{e}}(R)-F_{\bar{\nu}_{x}}(R)\right] \frac{R^{3}}{2 r^{3}} \\
& =3.5 \times 10^{5} \mathrm{~km}^{-1}\left(\frac{R}{r}\right)^{3}\left(\frac{L_{\bar{\nu}_{e}}}{\left\langle E_{\bar{\nu}_{e}}\right\rangle}-\frac{L_{\bar{\nu}_{x}}}{\left\langle E_{\bar{\nu}_{x}}\right\rangle}\right) \\
& \times \frac{15 \mathrm{MeV}}{10^{51} \mathrm{MeV} / \mathrm{s}}\left(\frac{10 \mathrm{~km}}{R}\right)^{2} .
\end{aligned}
$$

An equation analogous to Eq. (10) can be written for the anti-neutrinos.

One can define a conserved "lepton current" $\mathrm{L}^{\mu}=$ $\left(\mathrm{L}_{0}, \mathbf{L}\right)$ whose components are (see also [35])

$$
\begin{aligned}
& \mathrm{L}_{0}=\int_{0}^{1} d u^{\prime} \frac{1}{2}\left(\mathrm{D}_{+, u^{\prime}}+\mathrm{D}_{-, u^{\prime}}\right) \cdot \mathrm{B}, \\
& \mathrm{L}_{r}=\int_{0}^{1} d u^{\prime} v_{r} \frac{1}{2}\left(\mathrm{D}_{+, u^{\prime}}+\mathrm{D}_{-, u^{\prime}}\right) \cdot \mathrm{B}, \\
& \mathrm{L}_{t}=\int_{0}^{1} d u^{\prime}\left|v_{t}\right| \frac{1}{2}\left(\mathrm{D}_{+, u^{\prime}}-\mathrm{D}_{-, u^{\prime}}\right) \cdot \mathrm{B},
\end{aligned}
$$

where $\mathbf{L}$ is a two-dimensional vector $\left(\mathrm{L}_{r}, \mathrm{~L}_{\theta}\right)$, and $\mathrm{D}_{R(L), u} \cdot \mathrm{B} \simeq \mathrm{D}_{R(L), u}^{3}$. From Eq. (11) one realizes that the lepton current satisfies a continuity equation

$$
\partial_{0} \mathrm{~L}_{0}+\nabla_{\mathbf{r}} \cdot \mathbf{L}=\nabla_{\mathbf{r}} \cdot \mathbf{L}=0 \text {, }
$$

where first equality follows since $\partial_{0} \mathrm{~L}_{0}=0$ having we assumed a stationary solution. Eq. (19) generalizes the lepton-number conservation law of the one dimensional case [4].

\section{Equations of motion in Fourier space}

The differential operators in Eq. (9) implies that the flavor evolution is characterized by a partial differential equation problem in $r$ and $\phi$. In [29, 36] (see also [28]) it has been shown how it is possible to solve such a problem by Fourier transforming the equations of motion with respect to the coordinate along which a perturbation is introduced. We assume a perturbation of the polarization vectors at $r=R$ with period $2 \pi$ in $\phi$ so that

$$
\mathrm{P}_{ \pm, u}(R, \phi)=\mathrm{P}_{ \pm}^{0}+2 \mathrm{e}_{z} \delta \cos \phi
$$

where $\mathrm{P}_{ \pm}^{0}$ is the unperturbed value of the polarization vector, and $\delta \ll 1$ is the amplitude of the perturbation. Up to the small difference in the emission modes \pm [see Eq. (6)] the initial values of the polarization vectors are

$$
\begin{aligned}
& \mathrm{P}_{ \pm}^{0}(\nu) \simeq(1+\alpha) \mathrm{e}_{z}, \\
& \mathrm{P}_{ \pm}^{0}(\bar{\nu}) \simeq \mathrm{e}_{z},
\end{aligned}
$$

where the initial flavor asymmetry is given by

$$
\alpha=\frac{F_{\nu_{e}}-F_{\bar{\nu}_{e}}}{F_{\bar{\nu}_{e}}-F_{\bar{\nu}_{x}}} \text {. }
$$

The functions $\mathrm{P}_{ \pm, u}(r, \phi)$ are periodic in $\phi$ with period $2 \pi$. Their Fourier transform is then

$$
\mathrm{P}_{ \pm, u, n}(r)=\frac{1}{2 \pi} \int_{0}^{2 \pi} \mathrm{P}_{ \pm, u}(r, \phi) e^{-i n \phi} d \phi
$$

so that

$$
\mathrm{P}_{ \pm, u}(r, \phi)=\sum_{n=-\infty}^{+\infty} \mathrm{P}_{ \pm, u, n}(r) e^{+i n \phi} .
$$

The EoMs for the Fourier modes at large $r \gg R$ assume the form

$$
\begin{aligned}
& \frac{d}{d r} \mathrm{P}_{ \pm, u, n}(r)=\mp i n u \frac{R}{r^{2}} \mathrm{P}_{ \pm, u, n} \\
+ & \frac{\omega}{v_{r}} \mathrm{~B} \times \mathrm{P}_{ \pm, u, n} \\
+ & \mu_{r} \sum_{j=-\infty}^{+\infty} \int_{0}^{1} d u^{\prime}\left[\left(u^{2}+u^{\prime 2}\right) \frac{\left(\mathrm{D}_{+, u^{\prime}, n-j}+\mathrm{D}_{-, u^{\prime}, n-j}\right)}{2}\right. \\
& \left.\mp u u^{\prime}\left(\mathrm{D}_{+, u^{\prime}, n-j}-\mathrm{D}_{-, u^{\prime}, n-j}\right)\right] \times \mathrm{P}_{ \pm, u, j} .
\end{aligned}
$$

We stress that it is enough to follow the evolution for positive modes $n \geq 0$, since the $\mathrm{P}_{ \pm, u}(r, \phi)$ are real functions and therefore

$$
\mathrm{P}_{ \pm, u, n}^{*}=\mathrm{P}_{ \pm, u,-n}
$$

Once the evolution of the harmonic modes is obtained from Eq. (26), the polarization vector in configuration space can be obtained by inverse Fourier transform [Eq. (25)].

\section{NUMERICAL EXAMPLES}

We present the results of the flavor evolution in the two-dimensional model described above. To calculate the $\nu-\nu$ interaction strength in Eq. (15) and the flavor asymmetry parameter in Eq. 23 we use benchmark values often used in previous studies of self-induced neutrino oscillations (see, e.g. 14] ), i.e. we take as average energies

$$
\left(\left\langle E_{\nu_{e}}\right\rangle,\left\langle E_{\bar{\nu}_{e}}\right\rangle,\left\langle E_{\nu_{x}}\right\rangle\right)=(12,15,18) \mathrm{MeV}
$$

while for the neutrino luminosities (in units of $10^{51} \mathrm{erg} / \mathrm{s}$ ) we assume

$$
L_{\nu_{e}}=2.40 \quad, \quad L_{\bar{\nu}_{e}}=2.0 \quad, \quad L_{\nu_{x}}=1.50 .
$$

These values are typical of the early time SN accretion phase, and corresponds to an asymmetry parameter $\alpha=1.34$. As specified before we work in a single-energy 

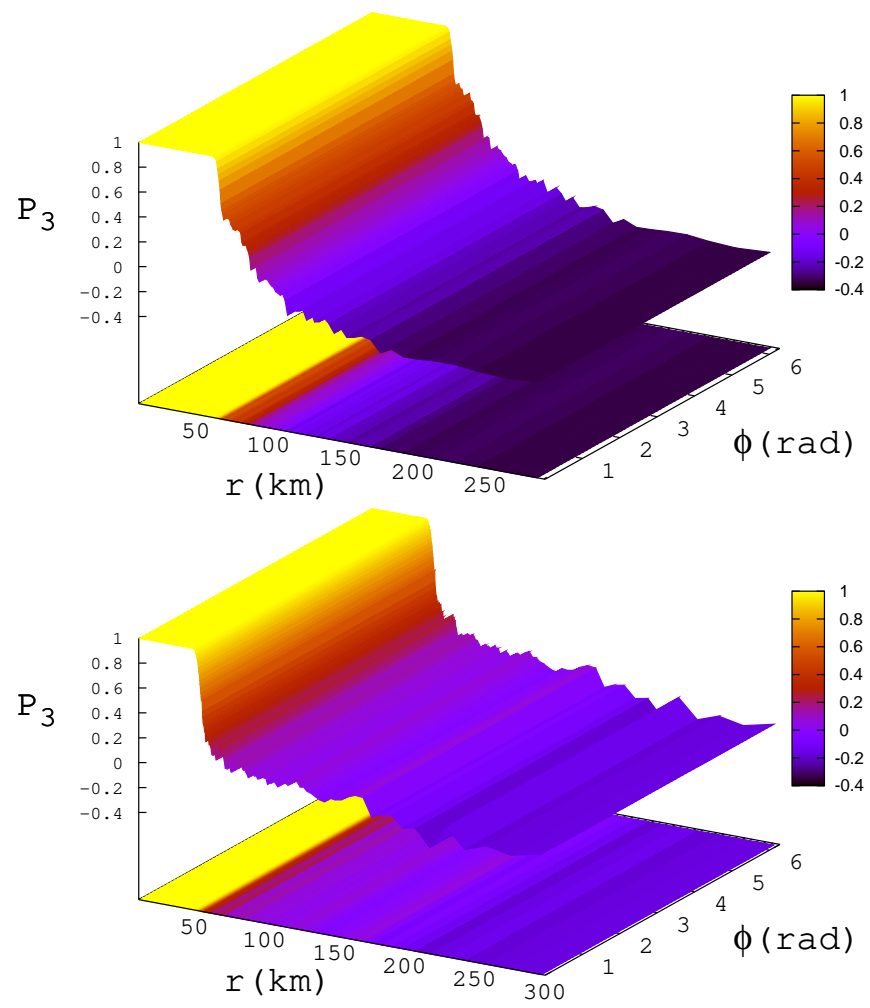

FIG. 2: Two-dimensional evolution of the 3-rd component $P_{3}$ of the $\bar{\nu}$ polarization vector in the $r-\phi$ plane, and its map on the bottom plane breaking only the \pm symmetry. Upper plot refers to $\mathrm{NH}$, while lower panel is for $\mathrm{IH}$.

scheme, where we take as representative vacuum oscillation frequency the one corresponding to the average of the $\nu$ ensemble with the emissivity parameters chosen above (see [14]). Namely we take $\omega=0.68 \mathrm{~km}^{-1}$. Concerning the neutrino oscillation parameters we choose a small mixing angle $\vartheta=10^{-2}$. Moreover, we assume $N_{u}=100$ modes for the angular variable $u \in[0 ; 1]$ in order to have numerical convergence of the results and to avoid spurious instabilities due to few angular modes.

It is known that forcing the \pm symmetry (taking $\beta_{+}=\beta_{-}=0$ in Eq. (6)) and the translational symmetry on the ring (taking $\delta=0$ in Eq. (20) ) the ensemble is stable in normal mass hierarchy (NH, $\Delta m^{2}>0$ ) while in inverted mass hierarchy $\left(\mathrm{IH}, \Delta m^{2}<0\right)$ it exhibits large bimodal flavor changes in the form of pair conversions $\nu_{e} \bar{\nu}_{e} \rightarrow \nu_{x} \bar{\nu}_{x}$ [4]. If we perturb the \pm symmetry taking small seeds $\beta_{+}=-\beta_{-}$in the distributions of Eq. (6) the system now exhibits the analogous of the so-called multi-azimuthal-angle (MAA) instability of the bulb model [21, 24, 25]. The result of the flavor evolution is shown in Fig. 2 where it is represented the behavior of the 3-rd component of the (integrated over $u$ ) antineutrino polarization vector $\mathrm{P}_{3}=1 / 2\left(\mathrm{P}_{+}+\mathrm{P}_{-}\right)$in the $(r, \phi)$ plane for $\mathrm{NH}$ (upper panel) and $\mathrm{IH}$ (lower panel). In these numerical examples we have chosen $\beta_{+}=10^{-2}$. The most striking effect of the MAA instability is that
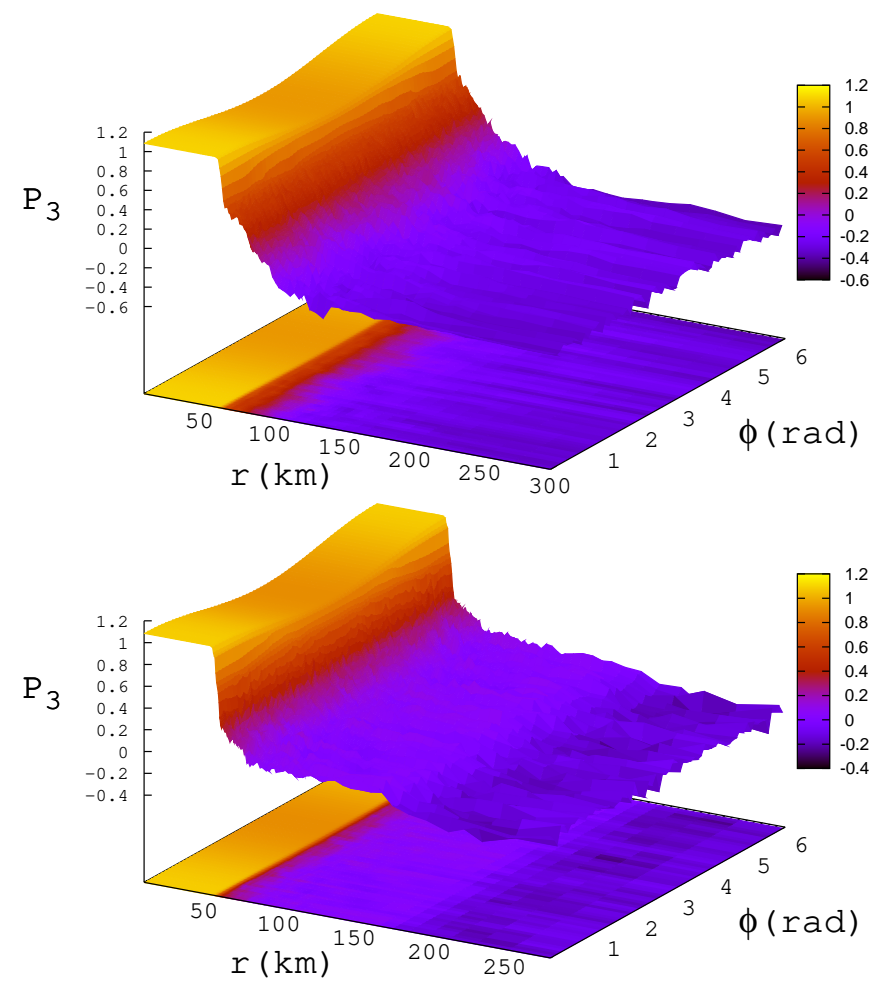

FIG. 3: Two-dimensional evolution of the 3-rd component $P_{3}$ of the $\bar{\nu}$ polarization vector in the $r$ - $\phi$ plane, and its map on the bottom plane breaking the azimuthal invariance for the \pm modes and the translational symmetry on the ring. Upper plot refers to $\mathrm{NH}$, while lower panel is for $\mathrm{IH}$.

now also NH exhibits flavor conversions at $r \gtrsim 60 \mathrm{~km}$. The choice of the initial seed $\beta_{ \pm}$determines the onset radius of the flavor conversions: the largest the seed, the earliest flavor conversions start. In IH (lower panel) flavor conversions start as in the \pm symmetric case at $r \gtrsim 50 \mathrm{~km}$ and MAA has a minor impact on the flavor evolution. From the Figure we realize that the behavior of the flavor conversions is uniform in the $\phi$ variable since the translational symmetry has remained unbroken. Indeed we have solved only the EoMs [Eq. (26)] for the $n=0$ Fourier mode in this case.

The next step is to perturb also the translational symmetry on the ring assuming a seed $\delta$ in the longitutudinal distribution of the polarization vectors on the boundary [see Eq. (20)]. In this case we consider the evoltion of the first $\mathrm{N}=100$ Fourier modes in Eq. (26). In this way we are sensitive to variations occuring at an angular scale $\Delta \phi \gtrsim 3^{\circ}$. Results are shown in Fig. 3 with the same format of the previous Figure. We used as seed to break the $\phi$-symmetry $\delta=3 \times 10^{-3}$. In both NH (upper panel) and IH case (lower panel) flavor conversions start as in the translational invariant case, i.e. the planes of common oscillation phase are flat in $\phi$ direction. However this behavior is not stable. In the NH case around $r \simeq 100 \mathrm{~km}$ something occurs: The $\mathrm{P}_{3}$ component is no longer flat in 


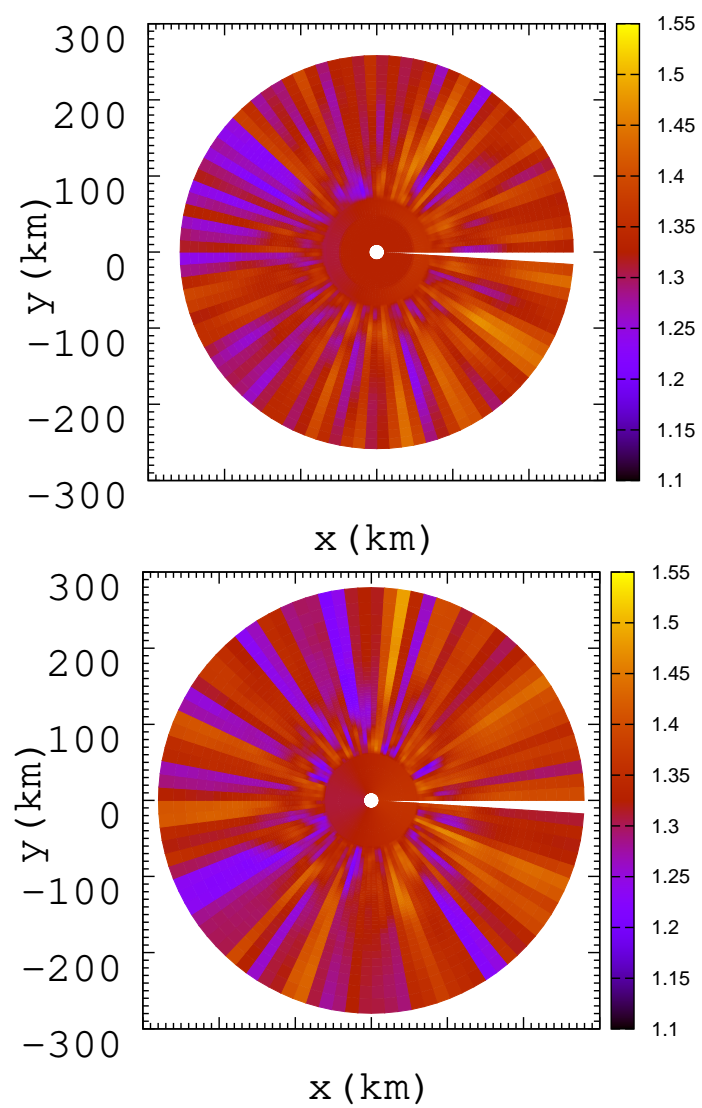

FIG. 4: Component $\mathrm{L}_{r}$ of the vector lepton number $\mathbf{L}$ in cartesian coordinates in NH (upper panel) and IH (lower panel), respectively.

$\phi$, while it starts to acquire notable variations $(\sim 20 \%)$ at different longitude. In IH after flavor conversions develop, also the translational symmetry is perturbed at $r \gtrsim 100 \mathrm{~km}$, with variations at different $\phi$ with values up to $30 \%$.

In Fig. 4 we represent the component $\mathrm{L}_{r}$ of the vector lepton number $\mathbf{L}$ [Eq. [18)] in cartesian coordinates

$$
\begin{aligned}
& x=r \cos \phi, \\
& y=r \sin \phi .
\end{aligned}
$$

We realize that when the spherical symmetry is broken, the lepton number acquires significant variations in different directions at a given $r$ with respect to the initial uniform value $\mathrm{L}_{r}=\alpha=1.34$. In particular one finds $\sim 20 \%$ variations.

In order to clarify better this flavor dynamics, in Fig. 5 we show a contour plot representing the growth of the different Fourier modes $\left|\mathrm{P}_{n}\right|$ (in logarithmic scale) in the plane $n-r$ for NH (upper panel) and IH (lower panel). We consider the evolution of the first $N=100$ modes. We realize that the breaking of the translational symmetry at $r \simeq 60 \mathrm{~km}$ in $\mathrm{NH}$ corresponds to the rapid excitation of the $n>0$ harmonics that reach values $\left|\mathrm{P}_{n}\right| \lesssim 10^{-2}$. Instead in $\mathrm{IH}$ for $r \gtrsim 50 \mathrm{~km}$ the modes start to get excited

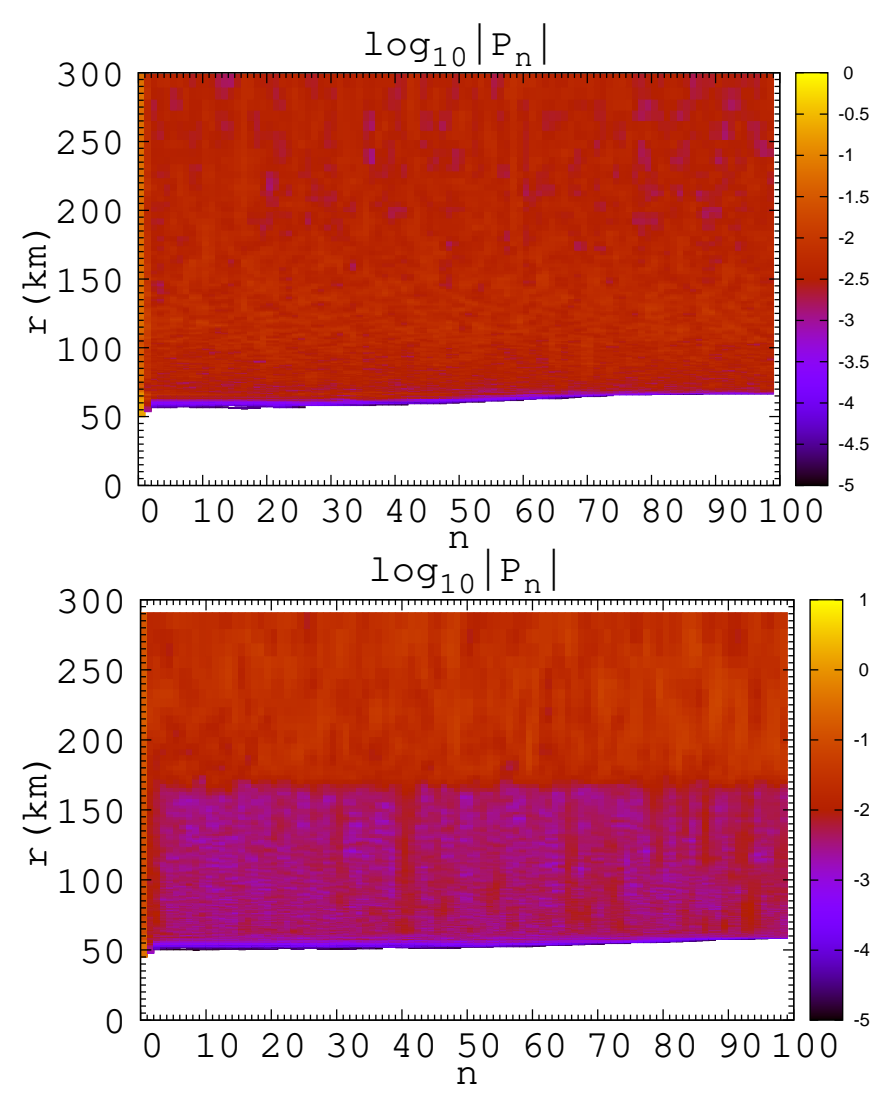

FIG. 5: Contour plots of the first 100 Fourier modes $\left|\mathrm{P}_{n}\right|$ (in logarithmic scale) in the plane $n-r$ in $\mathrm{NH}$ (upper panel) and IH (lower panel) respectively.

and can grow to $\left|\mathrm{P}_{n}\right| \gtrsim 10^{-1.5}$ at $r \gtrsim 150 \mathrm{~km}$. This explains why the effect of the breaking of the translational symmetry is more pronounced in $\mathrm{IH}$ rather than in $\mathrm{NH}$.

\section{CONCLUSIONS}

We have considered a simple two-dimensional toymodel, namely neutrino beams emitted from a ring, to point-out the effect of spontaneous breaking of axial and spherical symmetries in the self-induced flavor conversions of SN neutrinos.

We found that if the slightly perturb the space symmetries on the boundary, these perturbation seeds are dramatically amplified altering the flavor conversions found in a symmetric model. Therefore, the flavor content of the self-interacting SN neutrinos would acquire significant direction-dependent variations. These results are qualitatively similar to what we found in the planar model we studied in [14]. Our findings suggest that the characterization on the flavor conversions obtained before should be critically reconsidered, including these spontaneous symmetry breaking effects. In order to have a realistic characterization of the possible SN neutrino spectra our simple toy model should be improved on different aspects. In particular, one should extend this model 
to a realistic three-dimensional spherical case. In this case one would have the possibility to break the spherical symmetry in both longitudinal and latitudinal directions. Moreover, in order to get our numerical solution we have considered $N=100$ Fourier modes. In this cases we have not found the presence of flavor converions at lower radii than in the spherically symmetric case. However, in [28] it has been shown that harmonics with sufficiently high $n$ could become unstable also at low-radii. Increasing $N$ to 500 we have not found any sizeble change in the onset of the flavor changes and in the subsequent flavor evolution in the non-linear regime. However, it remains to be seen if with a much higher number of harmonics low-radii effects could occur. In this regard, it would be useful a stability analysis performed along the lines of [37].

Continuous energy spectra should also be taken into account to understand how the spectral splitting features found in the bulb model would be modified in this case. The role of matter effects that would suppress self-induced flavor conversions during the accretion phase should also be investigated. The final goal would be to study of the self-induced neutrino flavor con- versions in realistic multi-dimensional supernova models accounting for largely aspherical neutrino emission and matter profiles. This objective is particularly timely now since in the last recent years, SN model simulations have experienced several breakthroughs. After 1D [38] and 2D 33] models, the forefront has reached 3D SN simulations [39, 40]. Therefore, it seems the perfect juncture to connect realistic SN simulations with nonlinear neutrino oscillations. This open issue makes compulsory the need for further dedicated studies to fully clarify the fascinating behavior of the interacting neutrino field.

\section{Acknowledgements}

The author warmly thanks Pasquale Serpico for useful comments on this manuscript. This work is supported by the Italian Ministero dell'Istruzione, Università e Ricerca (MIUR) and Istituto Nazionale di Fisica Nucleare (INFN) through the "Theoretical Astroparticle Physics" projects.
[1] H. Duan, G. M. Fuller and Y. Z. Qian, "Collective Neutrino Oscillations," Ann. Rev. Nucl. Part. Sci. 60, 569 (2010) arXiv:1001.2799 [hep-ph]].

[2] H. Duan, G. M. Fuller and Y. Z. Qian, "Collective neutrino flavor transformation in supernovae," Phys. Rev. D 74, 123004 (2006) astro-ph/0511275.

[3] H. Duan, G. M. Fuller, J. Carlson and Y. -Z. Qian, "Simulation of Coherent Non-Linear Neutrino Flavor Transformation in the Supernova Environment. 1. Correlated Neutrino Trajectories," Phys. Rev. D 74, 105014 (2006) astro-ph/0606616.

[4] S. Hannestad, G. G. Raffelt, G. Sigl and Y. Y. Y. Wong, "Self-induced conversion in dense neutrino gases: Pendulum in flavour space," Phys. Rev. D 74, 105010 (2006) [Erratum-ibid. D 76, 029901 (2007)] astro-ph/0608695].

[5] G. L. Fogli, E. Lisi, A. Marrone and A. Mirizzi, "Collective neutrino flavor transitions in supernovae and the role of trajectory averaging," JCAP 0712, 010 (2007) arXiv:0707.1998 [hep-ph]].

[6] G. G. Raffelt and A. Y. Smirnov, "Self-induced spectral splits in supernova neutrino fluxes," Phys. Rev. D 76, 081301 (2007) [Phys. Rev. D 77, 029903 (2008)] arXiv:0705.1830 [hep-ph]].

[7] H. Duan, G. M. Fuller, J. Carlson and Y. Z. Qian, "Neutrino Mass Hierarchy and Stepwise Spectral Swapping of Supernova Neutrino Flavors," Phys. Rev. Lett. 99, 241802 (2007) arXiv:0707.0290 [astro-ph]].

[8] B. Dasgupta, A. Dighe, G. G. Raffelt and A. Y. .Smirnov, "Multiple Spectral Splits of Supernova Neutrinos," Phys. Rev. Lett. 103, 051105 (2009) arXiv:0904.3542 [hep$\mathrm{ph}]$.

[9] A. Friedland, "Self-refraction of supernova neutrinos: mixed spectra and three-flavor instabilities," Phys. Rev. Lett. 104, 191102 (2010) arXiv:1001.0996 [hep-ph]].

[10] B. Dasgupta, A. Mirizzi, I. Tamborra and R. Tomas,
"Neutrino mass hierarchy and three-flavor spectral splits of supernova neutrinos," Phys. Rev. D 81, 093008 (2010) arXiv:1002.2943 [hep-ph]].

[11] H. Duan, G. M. Fuller and Y. Z. Qian, "A Simple Picture for Neutrino Flavor Transformation in Supernovae," Phys. Rev. D 76, 085013 (2007) arXiv:0706.4293 [astro$\mathrm{ph}]$.

[12] Y. Z. Qian and G. M. Fuller, "Neutrino-neutrino scattering and matter enhanced neutrino flavor transformation in Supernovae," Phys. Rev. D 51, 1479 (1995) astro-ph/9406073.

[13] G. G. Raffelt and G. Sigl, "Self-induced decoherence in dense neutrino gases," Phys. Rev. D 75, 083002 (2007) hep-ph/0701182.

[14] A. Mirizzi and R. Tomas, "Multi-angle effects in selfinduced oscillations for different supernova neutrino fluxes," Phys. Rev. D 84, 033013 (2011) arXiv:1012.1339 [hep-ph]].

[15] R. F. Sawyer, "The multi-angle instability in dense neutrino systems," Phys. Rev. D 79, 105003 (2009) arXiv:0803.4319 [astro-ph]].

[16] A. Esteban-Pretel, S. Pastor, R. Tomàs, G. G. Raffelt and G. Sigl, "Decoherence in supernova neutrino transformations suppressed by deleptonization," Phys. Rev. D 76, 125018 (2007) arXiv:0706.2498 [astro-ph]].

[17] S. Chakraborty, T. Fischer, A. Mirizzi, N. Saviano and R. Tomas, "No collective neutrino flavor conversions during the supernova accretion phase," Phys. Rev. Lett. 107, 151101 (2011) arXiv:1104.4031 [hep-ph]].

[18] S. Chakraborty, T. Fischer, A. Mirizzi, N. Saviano and R. Tomas, "Analysis of matter suppression in collective neutrino oscillations during the supernova accretion phase," Phys. Rev. D 84, 025002 (2011) arXiv:1105.1130 [hep-ph]].

[19] N. Saviano, S. Chakraborty, T. Fischer and A. Mirizzi, 
"Stability analysis of collective neutrino oscillations in the supernova accretion phase with realistic energy and angle distributions," Phys. Rev. D 85, 113002 (2012) arXiv:1203.1484 [hep-ph]].

[20] S. Sarikas, G. G. Raffelt, L. Hudepohl and H. T. Janka, "Suppression of Self-Induced Flavor Conversion in the Supernova Accretion Phase," Phys. Rev. Lett. 108, 061101 (2012) arXiv:1109.3601 [astro-ph.SR]].

[21] G. Raffelt, S. Sarikas and D. de Sousa Seixas, "Axial Symmetry Breaking in Self-Induced Flavor Conversion of Supernova Neutrino Fluxes," Phys. Rev. Lett. 111, no. 9, 091101 (2013) [Erratum-ibid. 113, no. 23, 239903 (2014)] arXiv:1305.7140 [hep-ph]].

[22] G. Raffelt and D. d. S. Seixas, "Neutrino flavor pendulum in both mass hierarchies," Phys. Rev. D 88, 045031 (2013) arXiv:1307.7625 [hep-ph]].

[23] H. Duan, "Flavor Oscillation Modes In Dense Neutrino Media," Phys. Rev. D 88, 125008 (2013) arXiv:1309.7377 [hep-ph]].

[24] A. Mirizzi, "Multi-azimuthal-angle effects in self-induced supernova neutrino flavor conversions without axial symmetry," Phys. Rev. D 88, no. 7, 073004 (2013) arXiv:1308.1402 [hep-ph]].

[25] S. Chakraborty and A. Mirizzi, "Multi-azimuthal-angle instability for different supernova neutrino fluxes," Phys. Rev. D 90, no. 3, 033004 (2014) arXiv:1308.5255 [hep$\mathrm{ph}]$.

[26] S. Chakraborty, A. Mirizzi, N. Saviano and D. d. S. Seixas, "Suppression of the multi-azimuthalangle instability in dense neutrino gas during supernova accretion phase," Phys. Rev. D 89, no. 9, 093001 (2014) arXiv:1402.1767 [hep-ph]].

[27] S. Chakraborty, G. Raffelt, H. T. Janka and B. Mueller, "Supernova deleptonization asymmetry: Impact on selfinduced flavor conversion," arXiv:1412.0670 [hep-ph].

[28] H. Duan and S. Shalgar, "Flavor instabilities in the neutrino line model," Phys. Lett. B 747, 139 (2015) arXiv:1412.7097 [hep-ph]].

[29] A. Mirizzi, G. Mangano and N. Saviano, "Self-induced flavor instabilities of a dense neutrino stream in a twodimensional model," Phys. Rev. D 92, no. 2, 021702 (2015) arXiv:1503.03485 [hep-ph]].

[30] S. Sarikas, D. d. S. Seixas and G. Raffelt, "Spuri- ous instabilities in multi-angle simulations of collective flavor conversion," Phys. Rev. D 86, 125020 (2012) arXiv:1210.4557 [hep-ph]].

[31] G. Sigl and G. Raffelt, "General kinetic description of relativistic mixed neutrinos," Nucl. Phys. B 406, 423 (1993).

[32] P. Strack and A. Burrows, "Generalized Boltzmann formalism for oscillating neutrinos," Phys. Rev. D 71, 093004 (2005) hep-ph/0504035.

[33] R. Buras, M. Rampp, H.-T. Janka and K. Kifonidis, "Two-dimensional hydrodynamic core-collapse supernova simulations with spectral neutrino transport. 1 . Numerical method and results for a 15 solar mass star," Astron. Astrophys. 447, 1049 (2006) astro-ph/0507135.

[34] S. Abbar, H. Duan and S. Shalgar, "Flavor instabilities in the multiangle neutrino line model," Phys. Rev. D 92, no. 6, 065019 (2015) arXiv:1507.08992 [hep-ph]].

[35] H. Duan, G. M. Fuller and Y. Z. Qian, "Symmetries in collective neutrino oscillations," J. Phys. G 36, 105003 (2009) arXiv:0808.2046 [astro-ph]].

[36] G. Mangano, A. Mirizzi and N. Saviano, "Damping the neutrino flavor pendulum by breaking homogeneity," Phys. Rev. D 89, no. 7, 073017 (2014) arXiv:1403.1892 [hep-ph]].

[37] S. Chakraborty, R. S. Hansen, I. Izaguirre and G. Raffelt, "Self-induced flavor conversion of supernova neutrinos on small scales," arXiv:1507.07569 [hep-ph].

[38] T. Fischer, S. C. Whitehouse, A. Mezzacappa, F.K. Thielemann and M. Liebendorfer, "Protoneutron star evolution and the neutrino driven wind in general relativistic neutrino radiation hydrodynamics simulations," Astron. Astrophys. 517 (2010) A80 arXiv:0908.1871 [astro-ph.HE]].

[39] A. Wongwathanarat, E. Mueller and H.-T. Janka, "Three-Dimensional Simulations of Core-Collapse Supernovae: From Shock Revival to Shock Breakout," Astron. Astrophys. 577, A48 (2015) arXiv:1409.5431 [astroph.HE]].

[40] E. J. Lentz et al., "Three-dimensional Core-collapse Supernova Simulated Using a $15 M_{\odot}$ Progenitor," Astrophys. J. 807, 2, L31 (2015) arXiv:1505.05824 [astroph.HE]] 